Research Paper

\title{
Prognostic value of CEA/CA72-4 immunohisto- chemistry in combination with cytology for detecting tumor cells in peritoneal lavage in gastric cancer
}

\author{
Linlin $\mathrm{Qu}^{1}$, Liang $\mathrm{He}^{2}$, Zhifang Jia ${ }^{3}$, Quan Wang ${ }^{2 \bowtie}$ \\ 1. Department of Laboratory Medicine, the First Hospital of Jilin University, Jilin, China; \\ 2. Department of Gastrointestinal Surgery, the First Hospital of Jilin University, Jilin, China;
}

3. Department of Clinical Research, the First Hospital of Jilin University, Jilin, China.

$\square$ Corresponding author: Dr. Quan Wang, Department of Gastrointestinal Surgery, the First Hospital of Jilin University, Xinmin Street 71\#, Changchun 130021, Jilin, China. Tel: +86 15843073207, E-mail: wangquanjdyy@163.com

(c) The author(s). This is an open access article distributed under the terms of the Creative Commons Attribution License (https://creativecommons.org/licenses/by/4.0/). See http://ivyspring.com/terms for full terms and conditions.

Received: 2020.04.17; Accepted: 2020.08.14; Published: 2020.09.01

\begin{abstract}
Aim: To study the value and efficiency of CEA/CA72-4 immunohistochemistry in detecting free tumor cells from peritoneal lavage, in order to provide reliable lab information for subsequent intraperitoneal chemotherapy. Methods: A total of 112 progressive gastric cancer patients were enrolled from Oct. 2016 to Oct 2017, who were pathologically diagnosed as gastric cancer after surgery. Peritoneal lavage was respectively collected during operation. Cytology and CEA/CA72-4 immunohistochemistry of peritoneal lavage samples was performed. Overall survival and recurrence free survival was analyzed. Results: Cytology showed 16 positive cases (14.29\%), CEA immunohistochemistry showed 29 positive cases (25.89\%), CA72-4 immunohistochemistry showed 33 positive cases $(29.46 \%)$. McNemar's test showed significant difference in positivity between cytology $(\mathrm{CY}+)$ and CEA/CA72-4 immunohistochemistry (IHC+). Kappa test showed consistency between immunohistochemistry of CEA and CA72-4 with cytology. Patients with $\mathrm{CY}+/ \mathrm{IHC}+$ had the poorest overall survival (OS) as well as recurrence free survival (RFS), followed by those with $\mathrm{CY}+$ or $\mathrm{IHC}+$, while those with $\mathrm{CY}-/ \mathrm{IHC}$ - had higher OS and RFS. The differences of OS and RFS in IHC+ group were worse than that in IHC- group. Kaplan-Meier analysis showed that positive CEA/CA72-4 IHC revealed poorer prognosis than the negative cases. Conclusions: Due to the limitation of cytology, combination of cytology and immunohistochemistry appears to be more efficient for predicting prognosis of progressive gastric cancer.
\end{abstract}

Key words: Gastric Cancer; Peritoneal Lavage; Metastasis; Prognosis; Immunohistochemistry.

\section{INTRODUCTION}

In recent years, mortality of gastric cancer has reduced due to radical gastrectomy accompanied by adjuvant chemotherapy. However, in worldwide, gastric cancer is still the third leading cause of cancer death [1]. Clinical decisions of post-adjuvant treatment for gastric cancers are mainly depended on tumor invasive depth, TNM staging, and whether lymph nodes metastasis and/or peritoneal metastasis or not [2]. Thus, besides traditional pathology, the detection of free tumor cells in peritoneal lavage appears to be very important in post-adjuvant decision making for those advanced gastric cancers.

The aim of this study is to confirm the presence of the small amount of free tumor cells in peritoneal lavage, to evaluate methods of detection for these cells, and to explore the value of detecting free tumor cells in prognosis and post-adjuvant decision making.

\section{MATERIALS AND METHODS}

\section{Patients and Clinical Treatments}

In this study, 112 patients with advanced GC (GC: gastric cancer) who underwent open or 
laparoscopic gastrectomy as first treatment in the First Hospital of Jilin University from Oct. 2016 - Oct. 2017 were included in the study, who were pathologically diagnosed and other tumors were excluded. All the patients had radical gastrectomy of gastric cancer with pathology-proven negative cutting edges.

According to the seventh AJCC (AJCC: American Joint Committee on Cancer)/International Union Against Cancer, TNM system [3] was used for the staging of gastric cancer.

In GC group, patients with positive cytological findings received intraperitoneal chemotherapy after surgery. Paclitaxel was injected intravenously (50 $\mathrm{mg} / \mathrm{m}^{2}$ ) on day 1 and intraperitoneally (diluted in 1.0 $\mathrm{L}$ of normal saline, $\left.20 \mathrm{mg} / \mathrm{m}^{2}\right)$ on day 8 . Take S-1 (80 $\mathrm{mg} / \mathrm{m}^{2}$ ) orally, daily (1-14 days, rest for 1 week). Treatment was repeated every 3 weeks until the disease progress or intolerable toxicity occurred.

\section{Ethical Declaration}

This study was performed according to the Declaration of Helsinki laid down in 1964 and was approved by the Ethics Committee of the First Hospital of Jilin University. Patients were informed before operation and voluntarily participated in the study. Privacy of patients was conserved.

\section{Peritoneal Lavage Collection}

At the first time when laparoscopic puncture was set into the peritoneal cavity, wash gastric bed, spleen fossa and Douglas pouch with $500 \mathrm{ml}$ of warm saline, and then aspirated out to a clean container, which was designated as the first PLs (PLs: peritoneal lavage samples). Drain directly for those who already had ascites. After the complete removal of the tumor or lesion, the peritoneal cavity was washed and aspirated. PL samples were centrifuged under $1500 \mathrm{rpm}, 5 \mathrm{~min}$ at room temperature to collect intact cells. The precipitates were used for cytology and immunohistochemistry.

\section{Pathology and Cytology Examination}

After surgery, the tumors underwent pathological examination. HE staining was used on the slides from the paraffin blocks of each sample. The precipitates of PLs were smeared onto several slides by Autoslide Slide Maker Stainer (Simens, SN ABX15091316). Cytology was done followed by Pap staining using hematoxylin (Sigma, CAS 517-28-2). A positive result for cytology was marked as CY+ (Figure $1 \mathrm{~A}$ ), while CY- for a negative cytology result.

\section{Immunohistochemistry Staining and Evaluation}

IHC (IHC: Immunohistochemistry) was used to examine the expression of CEA and CA72-4 in tumor cells from PLs. A cytologically positive slides was taken as positive control (Figure $1 \mathrm{~B} / \mathrm{C}$ ), and a non-cancer PL sample was taken as negative control. Slides of PLs were fixed with $4 \%$ paraformaldehyde, then put in $3 \% \quad \mathrm{H}_{2} \mathrm{O}_{2}$ to exhaust endogenous peroxidase; slides were incubated overnight in $4^{\circ} \mathrm{C}$ with primary antibodies, rabbit monoclonal anti-CEA (Abcam, Cambridge, MA) and rabbit monoclonal anti-CA72-4 (Abcam, Cambridge, MA), and then goat anti-rabbit IgG secondary antibodies (Abcam, Cambridge, MA) for $1 \mathrm{hr}$. After incubation with the appropriate HRP (HRP: horseradish peroxidase) conjugated antibodies (Bioworld Technology, St. Louis Park, MN) for 2 hours, the signals were observed with a Diaminobenzidine (DAB) kit, stained with hematoxylin. The IHC slides were observed through microscope.

Cytoplamic CEA and CA72-4 expression inside free cancer cells of PLs were evaluated and scored using microscopy. By assessing the proportion of positive cells among the whole cells, scores were recorded as A: $0(<5 \%), 1(6-25 \%), 2(26-50 \%), 3$ $(51-75 \%), 4$ (76-100\%); the intensity of staining were graded as B: 0 (negative), 1 (weak staining), 2 (medium staining), 3 (strong staining). The final score for each sample was calculated by average $\mathrm{A} \times \mathrm{B}$ of successive 10 high power fields. A final score $>1$ was considered as positive and marked as IHC+, otherwise marked as IHC-.

\section{Follow-up}

GC patients were followed up 2.4-24 months after surgery until death or the scheduled deadline. Examination for patients included CT scan and gastroscopy. CT scan and gastroscopy was performed every six months. All CT images were validated by an experienced radiologist who was informed of the patients' pathological diagnosis but blinded to the disease progression. Symptoms of peritoneal metastasis within 6 months after surgery were all regarded as peritoneal metastasis before surgery. Patients with positive cytology during surgery or peritoneal metastasis within 6 months after surgery were all classified as the peritoneal metastasis group, which was used as the golden standard to measure the value of immunohistochemistry. Rates of true positive, true negative, false positive and false negative were recorded. Gastroscopy results were provided by an experienced physicist. CR (CR: complete response), PR (PR: partial response), SD (SD: stable disease), and PD (SD: stable disease) were assessed according to the RECIST Criteria: version 1.1 [4]. 


\section{Statistical Analysis}

By SPSS 23.0 software, the positive rates of immunohistochemistry and cytology were compared using McNemar's test. Kappa coefficient was used to measure the consistency of results from CEA/CA72-4 IHC and cytology. The sensitivity and specificity to identifying peritoneal metastasis were calculated. The survival curves were plot using Kaplan-Meier method for both OS (OS: overall survival) and RFS (RFS: recurrence free survival), and then compared using log-rank test. Statistical significance was considered as two-sided $P$ value $<0.05$.

\section{RESULTS}

\section{Patients' Information}

The clinical and pathological information of the patients was summarized in Table 1.

Table 1. Basic information and pathological features of GC patients $(\mathrm{N}=112)$.

\begin{tabular}{|c|c|c|}
\hline Characteristics & Classification & Number of cases (\%) \\
\hline \multirow[t]{2}{*}{ Age } & $\geq 60$ & $60(53.57)$ \\
\hline & $<60$ & $52(46.43)$ \\
\hline \multirow[t]{2}{*}{ Gender } & Male & $93(83.04)$ \\
\hline & Female & $19(16.96)$ \\
\hline \multirow[t]{2}{*}{ Tumor location } & Fundus ventriculi & $24(21.43)$ \\
\hline & $\begin{array}{l}\text { Gastric angle/ } \\
\text { Gastric antrum }\end{array}$ & $88(78.57)$ \\
\hline \multirow[t]{2}{*}{ Tumor size } & $\geq 5 \mathrm{~cm}$ & $59(52.68)$ \\
\hline & $<5 \mathrm{~cm}$ & $53(47.32)$ \\
\hline \multirow[t]{4}{*}{ Histology } & Low differentiated & $49(43.75)$ \\
\hline & $\begin{array}{l}\text { Low-moderate } \\
\text { differentiated }\end{array}$ & $46(41.07)$ \\
\hline & Moderate differentiated & $12(10.71)$ \\
\hline & $\begin{array}{l}\text { Signet ring cell } \\
\text { carcinoma }\end{array}$ & $5(4.47)$ \\
\hline \multirow[t]{3}{*}{ Lauren classification } & Intestinal-type & $21(18.75)$ \\
\hline & Mixed-type & $40(35.71)$ \\
\hline & Diffused-type & $51(45.54)$ \\
\hline \multirow[t]{2}{*}{ Vascular/ neural invasion } & Yes & $106(94.64)$ \\
\hline & No & $6(5.36)$ \\
\hline \multirow[t]{3}{*}{ Depth of invasion } & T1/T2 & $6(5.36)$ \\
\hline & $\mathrm{T} 3$ & $103(90.18)$ \\
\hline & $\mathrm{T} 4$ & $3(2.68)$ \\
\hline \multirow[t]{2}{*}{ Lymph node metastasis } & Yes & $104(92.86)$ \\
\hline & No & $8(7.14)$ \\
\hline
\end{tabular}

\section{Positivity and Consistency of CEA/CA72-4 Cytology and Immunohistochemistry}

The positive control of cytology and CEA/CA72-4 immunohistochemistry showed in Figure 1. Among the 112 cases, CEA immunohistochemistry showed 29 positive cases $(25.89 \%)$ and 83 negative cases (74.11\%), while CA72-4 immunohistochemistry showed 33 positive cases $(29.46 \%)$ and 89 negative cases (79.46\%) (Table 2). McNemar's test showed no significant difference between CEA and CA72-4 immunohistochemistry $(p=0.219)$. Cytology showed 16 positive cases $(14.29 \%)$ and 96 negative cases $(85.71 \%)$, among which there were 18 immunochemistry $(+)$ cases showing cytology $(-)$. The total positive rate of immunohistochemistry was $30.36 \% \quad(34 / 112)$ (Table 3). Positivity of immunohistochemistry was higher than cytology, and McNemar's test showed significant difference in positivity between cytology and immunohistochemistry $(p<0.001)$. Kappa test showed consistency between immunohistochemistry with cytology was $0.866(\mathrm{p}<0.001)$.

Table 2. Results of CEA and CA72-4 immunohistochemistry examination $(\mathrm{N}=112)$.

\begin{tabular}{llll}
\hline CA72-4 & \multicolumn{2}{l}{ CEA Immunohistochemistry } & Total (n/\%) \\
\cline { 2 - 3 } Immunohistochemistry & positive & negative & \\
\hline positive & 28 & 5 & $33 / 29.46 \%$ \\
negative & 1 & 78 & $79 / 70.54 \%$ \\
Total (n/\%) & $29 / 25.89 \%$ & $83 / 74.11 \%$ & $112 / 100 \%$ \\
\hline
\end{tabular}

Table 3. Results of immunohistochemistry and cytology $(N=112)$.

\begin{tabular}{llll}
\hline Immunohistochemistry & \multicolumn{2}{l}{ Cytology } & Total $(\mathrm{n} / \%)$ \\
\cline { 2 - 3 } & positive & negative & \\
\hline positive & 16 & 18 & $34 / 30.36 \%$ \\
negative & 0 & 78 & $78 / 69.64 \%$ \\
Total (n/\%) & $16 / 14.29 \%$ & $96 / 85.71 \%$ & $112 / 100 \%$ \\
\hline
\end{tabular}

\section{Sensitivity and Specificity of Cytology and Immunohistochemistry}

In this study, peritoneal metastasis defined as positive cytology or peritoneal metastasis in 6 months after surgery. Until the end of follow up, there were 59 cases with peritoneal metastasis. True positive, true negative, false positive, false negative of cytology and immunohistochemistry were list in Table 4 . Sensitivity, specificity and Youden index were calculated (Table 4).

Table 4. Results of cytology and immunohistochemistry in peritoneal metastasis $(\mathrm{N}=112)$.

\begin{tabular}{llllll}
\hline Methods/ Results & \multicolumn{2}{l}{$\begin{array}{l}\text { Peritoneal } \\
\text { metastasis }\end{array}$} & $\begin{array}{l}\text { Sensitivity } \\
(\%)\end{array}$ & $\begin{array}{l}\text { Specificity } \\
(\%)\end{array}$ & $\begin{array}{l}\text { Youden } \\
\text { index }\end{array}$ \\
\cline { 2 - 4 } & Positive & Negative & & \\
\hline $\begin{array}{lllll}\text { Cytology } \\
\text { Positive }\end{array}$ & 16 & 0 & $59.26 \%$ & $100.00 \%$ & 0.6 \\
Negative & 11 & 85 & & & \\
Immunohistochemistry & 27 & 7 & $100.00 \%$ & $91.76 \%$ & 0.9 \\
Positive & 0 & 78 & & & \\
Negative & 27 & 85 & & & \\
Total (n) & & & & & \\
\hline
\end{tabular}




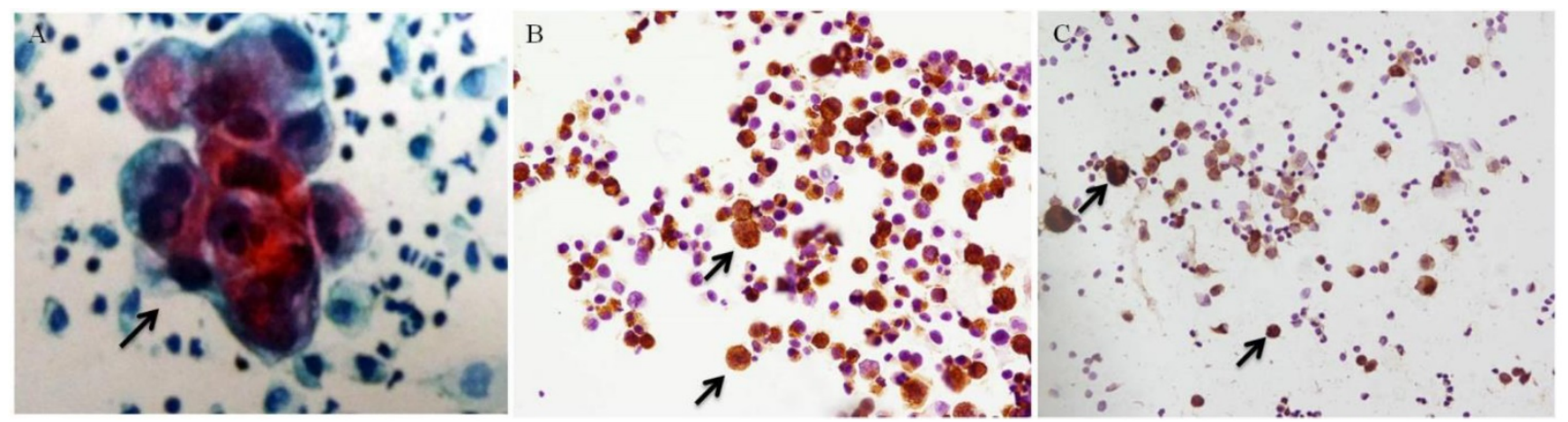

Figure 1. Positive control of cytology and immunohistochemistry (black arrows, $\times 200$ ). (A) Positive cytology under Pap staining. (B) Positive CA72-4 immunohistochemistry. (C) Positive CEA immunohistochemistry.
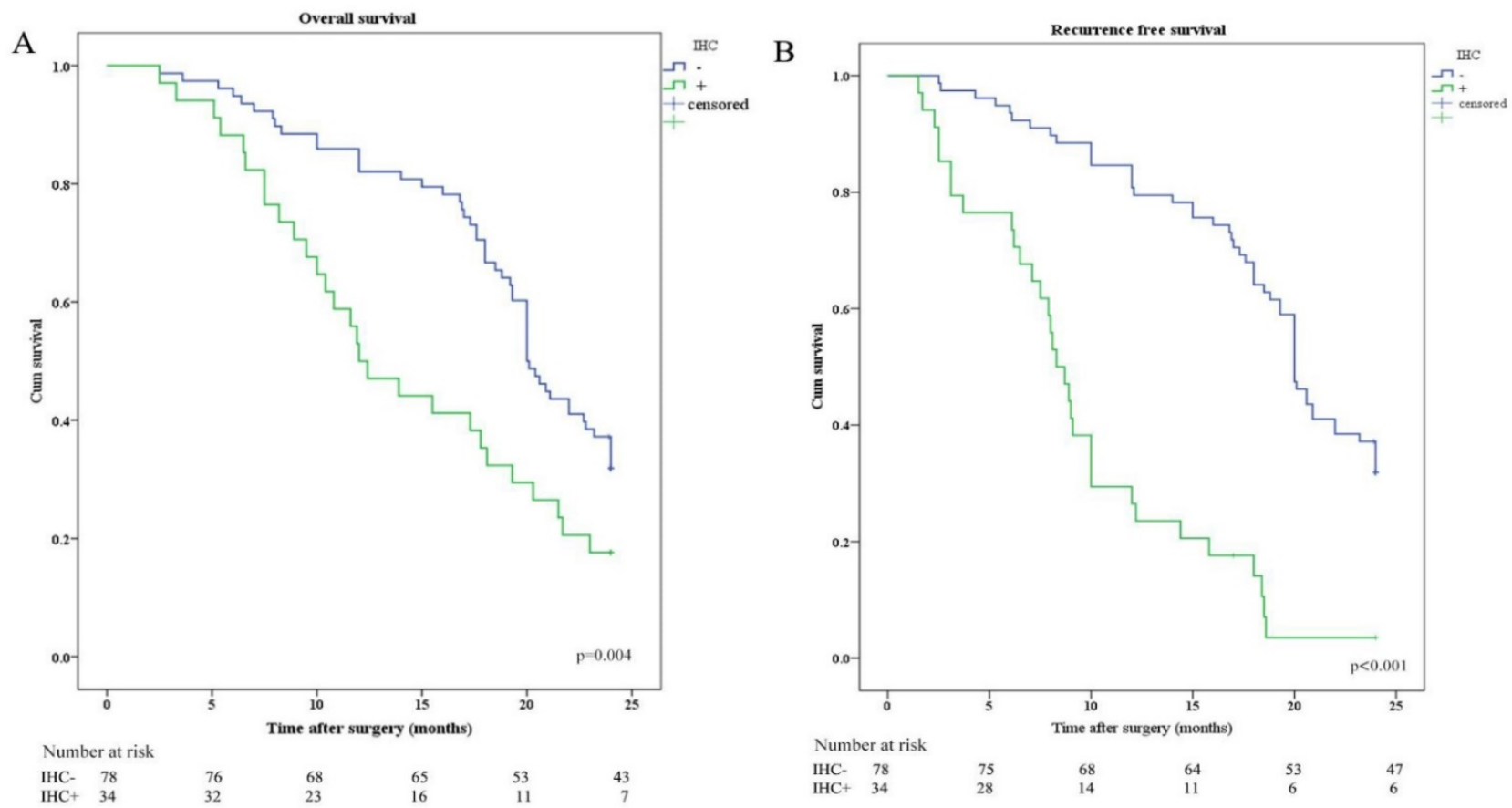

Figure 2. Kaplan-Meier analysis of OS and RFS stratified by IHC results. (A) Kaplan-Meier analysis of OS between IHC positive (IHC+) and IHC negative (IHC-). (B) Kaplan-Meier analysis of RFS between IHC positive (IHC+) and IHC negative (IHC-).

\section{Survival Analysis Cytology and Immunohistochemistry}

OS and RFS were plotted according to CY (CY: cytology) and IHC of peritoneal lavage. Median OS in patients with CY-/IHC-, CY+ or IHC+, and $\mathrm{CY}+/ \mathrm{IHC}+$ were 20.0 months, 12.0 months, and 10.8 months, respectively. In order to investigate the relationship between IHC and survival, the differences in OS and RFS between IHC+ and IHCpatients were analyzed and revealed that OS and RFS were worse in IHC+ group (Figure 2). The relationship between $\mathrm{CY}$ and survival was also analyzed, revealing that OS and RFS were worse in $\mathrm{CY}+$ group (Figure 3). Patients with $\mathrm{CY}+/ \mathrm{IHC}+$ ("double positive") had the poorest OS as well as RFS, followed by those with $\mathrm{CY}+$ or $\mathrm{IHC}+($ "single positive"), while those with CY-/IHC-("double negative"), had higher OS and RFS, with statistically significant differences (Figure 4).

\section{DISCUSSION}

GC is the fifth most common malignant cancer and the third leading cause of cancer death in the world [5]. During 2008-2014, 5-year relative survival in the US is $31.0 \%$ for all stages, $68.1 \%$ for localized tumors, $30.6 \%$ for regional tumors, and $5.2 \%$ for distant tumors [6], which suggests that distant metastasis is the main threat to deaths in gastric cancer. Among distant metastasis, peritoneal metastasis is the most common in gastric cancer [7]. The updated TNM Staging by AJCC (AJCC: American Joint Committee on Cancer) had classified peritoneal metastasis as M1c [8, 9], positive peritoneal lavage cytology is defined as M1 for staging by the Stomach Cancer Group of the Japan Clinical Oncology Group 
(JCOG) [10]. Although cytological examination of peritoneal lavage is considered as a traditional method for detecting free tumor cells in peritoneal lavage [11], it is reported that detection rates were $2.2 \% \sim 47.2 \%$ and more than half of positive cases were missed due to the limitations in the aspect of sensitivity. In recent years, some independent studies confirmed that intraperitoneal treatment strategy is necessary for the peritoneal metastatic cases [12, 13]. The sensitivity of recognition for peritoneal metastasis can significantly improve patients' prognosis [14, 15]. The need for precise diagnosis of metastasis lies in IP (IP: intraperitoneal chemotherapy) decision making, for example, a meta-analysis was carried out which showed IP can benefit patients' prognosis [16]. Patients with gastric cancer under IP had a higher rate of negative lavage cytology [17]. Thus, finding novel and useful biomarkers to improve the efficiency for detecting peritoneal metastasis is very important [18]. Beside peritoneal cytology, genetic detection of CEA [19], CK20 (CK20: cytokeratin 20) [20], CK19 (CK19: cytokeratin 19) [21], and MAGE (MAGE: melanoma-associated gene) [22] using RT-PCR (RT-PCR: reverse transcriptase-polymerase chain reaction) were reported as one of available tools that correlated with patients' metastasis. Moreover, immunohistochemistry of cells from peritoneal lavage is considered another available strategy for directly detecting metastatic tumor cells.

In this study, we chose CEA and CA72-4 as candidate markers for immunochemistry examination to detect free tumor cells from peritoneal lavage for patients with gastric cancer. CEA expression in peritoneal lavage fluid was reported to have significantly poorer OS and RFS compared with those negative cases [7]. Several evidence indicated that CEA was the most sensitive biomarker for analyzing gastric cancer $[8,9]$. However, the specificity of CEA is insufficient because it is widely synthesized and secreted by many tumors such as ovarian cancer, pancreatic carcinoma, colorectal cancer, and lung cancer. Yet there were no convincing partner markers as compensate for the weakness of CEA. Therefore, we tried to evaluate the efficiency of CA72-4 in the establishment of immunohistochemistry examination.

From the above findings, we conclude that CEA/CA72-4 immunohistochemistry may become a new method to evaluate peritoneal metastasis as an improvement for the limitations of cytology. The contribution of the study is to establish an alternative tool to predict peritoneal metastasis for patients with gastric cancer. Due to the feasibility of peritoneal lavage during radical gastrectomy, the application of CEA/CA72-4 immunohistochemistry emerges as a more sensitive indicator to predict the prognosis of these patients, as well as the timely decision making of post-adjuvant therapy. Objectively, this study has some limitations regarding on the relatively small sample size, heterogeneity of postoperative treatment. Further, a large-scale observation will be required to determine whether this examination can be widely adopted in the clinic.
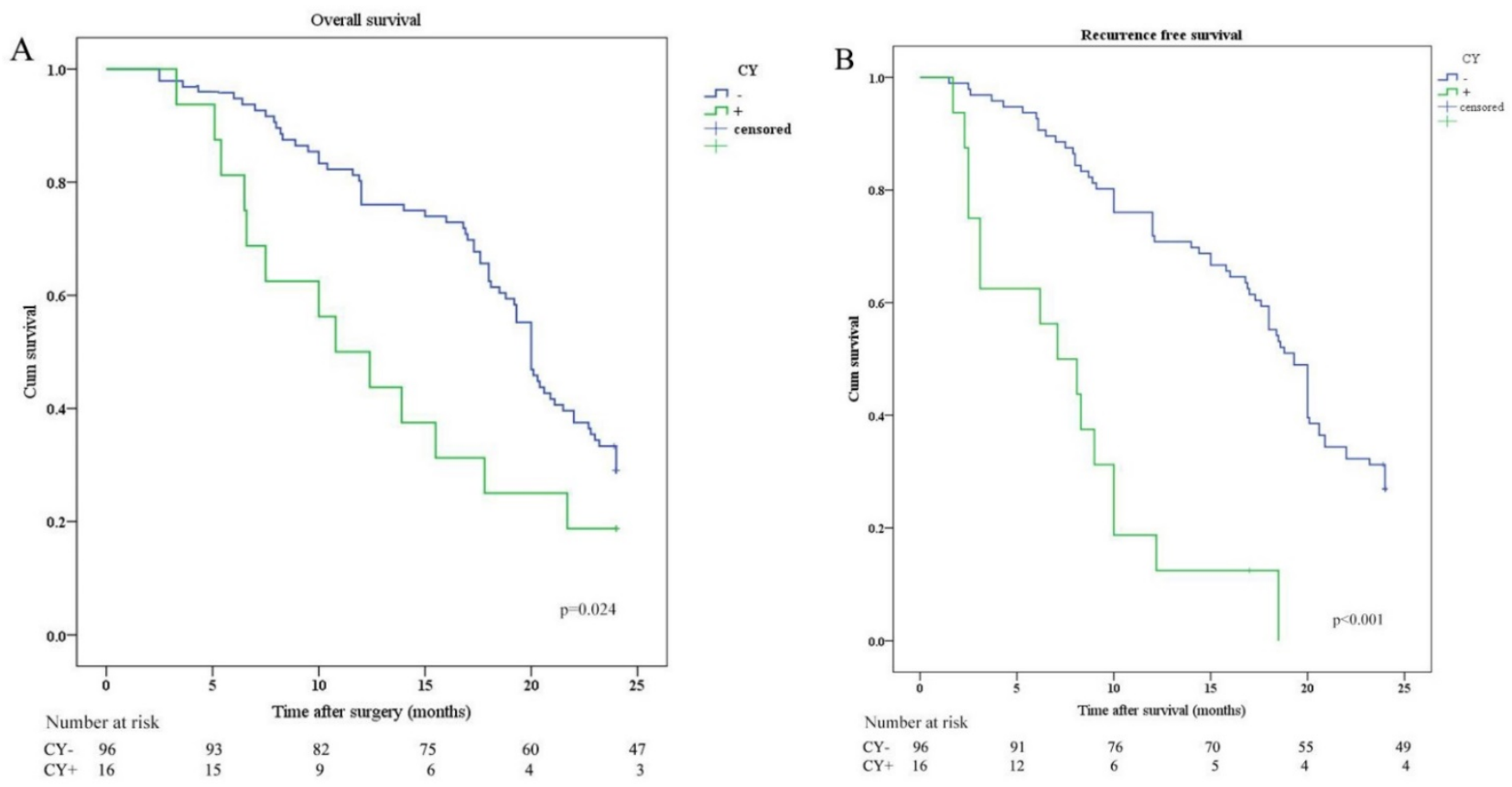

Figure 3. Kaplan-Meier analysis of OS and RFS stratified by CY results. (A) Kaplan-Meier analysis of OS between CY positive (CY+) and CY negative (CY-). (B) Kaplan-Meier analysis of RFS between $\mathrm{CY}$ positive $(\mathrm{CY}+)$ and $\mathrm{CY}$ negative (CY-). 

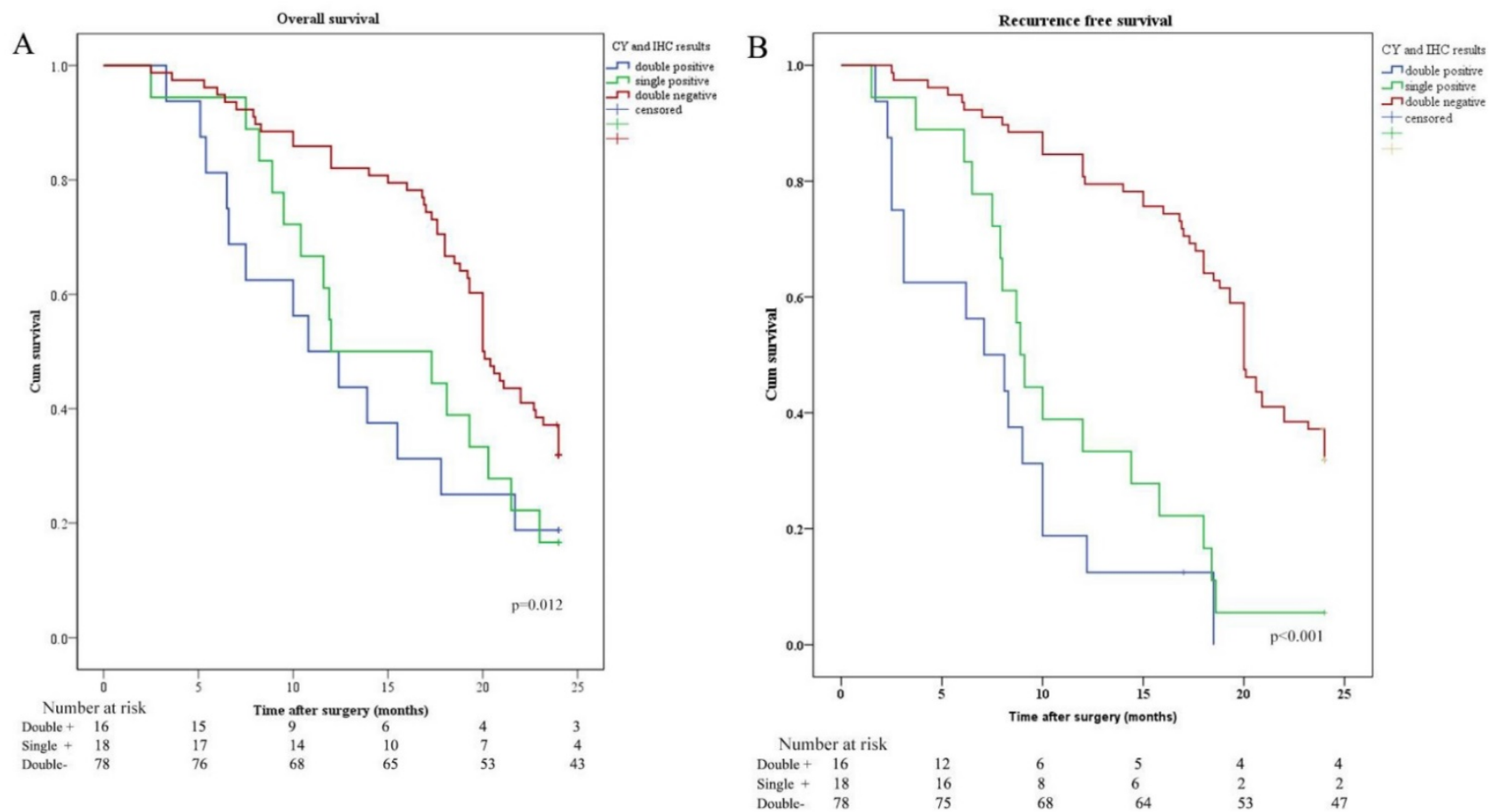

Figure 4. Kaplan-Meier analysis of OS and RFS stratified by $\mathrm{CY}$ and IHC results. (A) Kaplan-Meier analysis of OS between $\mathrm{CY}$ and IHC double positive (CY+/IHC+), single positive (CY+ or $\mathrm{IHC}+$ ), and double negative (CY-/IHC-). (B) Kaplan-Meier analysis of RFS among CY+/lHC+, CY+ or IHC+, and CY-//HC-.

\section{ACKNOWLEDGEMENT}

This work was partially supported by Department of Science and technology of Jilin Province, as an application extension of our previous basic research supported by Fund No.20170520011JH; as a technological attempt to detect peritoneal metastasis, this work was partially supported by Transformation Department of the First Hospital of Jilin University Fund No. BQEGCZX2019006.

\section{Ethical approval}

All procedures performed in studies involving human participants were in accordance with the ethical standards of the institutional and national research committee and with the 1964 Helsinki declaration and its later amendments or comparable ethical standards.

\section{Informed consent}

Informed consent was approved by the Clinical Research Ethics Committee of the First Hospital of Jilin University.

\section{Key messages}

Detecting cast-off tumor cells in peritoneal lavage of gastric cancer patients can provide more accurate clinical information and the following decision making. In this article, we reported that CEA and CA72-4 immunohistochemistry had higher positivity than cytology in detecting tumor cells from peritoneal lavage of gastric cancer. Moreover, CEA/CA72-4 immunohistochemistry showed consistency with cytology, and poorer overall survival as well as recurrence free survival in those with positive immunohistochemistry results. These findings suggest that CEA/CA72-4 immunohistochemistry may have prognostic value for gastric cancer patients and help doctor in decision making.

\section{Competing Interests}

The authors have declared that no competing interest exists.

\section{References}

1. Torre LA, Bray F, Siegel RL, Ferlay J, Lortet-Tieulent J, Jemal A. Global cancer statistics, 2012. CA: a cancer journal for clinicians. 2015; 65: 87-108.

2. Kanaji S, Suzuki S, Matsuda Y, Hasegawa H, Yamamoto M, Yamashita K, et al. Recent updates in perioperative chemotherapy and recurrence pattern of gastric cancer. Annals of gastroenterological surgery. 2018; 2: 400-5.

3. Zhang J, Niu Z, Zhou Y, Cao S. A comparison between the seventh and sixth editions of the American Joint Committee on Cancer/International Union Against classification of gastric cancer. Annals of surgery. 2013; 257: 81-6.

4. Eisenhauer EA, Therasse P, Bogaerts I, Schwartz LH, Sargent D, Ford R, et al. New response evaluation criteria in solid tumours: revised RECIST guideline (version 1.1). European journal of cancer. 2009; 45: 228-47.

5. Bray F, Ferlay J, Soeriomataram I, Siegel RL, Torre LA, Jemal A. Global cancer statistics 2018: GLOBOCAN estimates of incidence and mortality worldwide for 36 cancers in 185 countries. CA: a cancer journal for clinicians. 2018; 68: 394-424.

6. Noone AM HN, Krapcho M, et al. SEER Cancer Statistics Review, 1975- 2015. Bethesda, MD, https://seercancergov/csr/1975-2015/, based on November 2017 SEER data submission, posted to the SEER web site, National Cancer Institute. 2018.

7. Ji L, Selleck MJ, Morgan JW, Xu J, Babcock BD, Shavlik D, et al. Gastric Cancer Peritoneal Carcinomatosis Risk Score. Annals of surgical oncology. 2020; 27: $240-7$. 
8. Jessup JM GR, Aware EA, et al. Colon and Rectum. In: AJCC Cancer Staging Manual, 8th, Amin MB (Ed), AJCC, Chicago. 2017; p.251.

9. Wittekind C. The development of the TNM classification of gastric cancer. Pathology international. 2015; 65: 399-403.

10. Japanese Gastric Cancer A. Japanese classification of gastric carcinoma: 3rd English edition. Gastric cancer: official journal of the International Gastric Cancer Association and the Japanese Gastric Cancer Association. 2011; 14: 101-12.

11. Burke EC, Karpeh MS, Jr., Conlon KC, Brennan MF. Peritoneal lavage cytology in gastric cancer: an independent predictor of outcome. Annals of surgical oncology. 1998; 5: 411-5.

12. Kano Y, Kosugi SI, Ishikawa T, Otani T, Wakai T. Prognostic significance of peritoneal lavage cytology at three cavities in patients with gastric cancer. Surgery. 2015; 158: 1581-9.

13. Nakayama I, Chin K, Matsushima T, Takahari D, Ogura M, Shinozaki E, et al. Retrospective comparison of S-1 plus cisplatin versus S-1 monotherapy for the treatment of advanced gastric cancer patients with positive peritoneal cytology but without gross peritoneal metastasis. International journal of clinical oncology. 2017; 22: 1060-8.

14. Liang H. [The Precised Management of Surgical Treatment for Gastric Cancer: Interpretation of the 5th edition of Japanese Gastric Cancer Treatment Guideline and the 15th edition of Japanese Classification for Gastric Cancer]. Zhonghua zhong liu za zhi [Chinese journal of oncology]. 2019; 41: 168-72.

15. Yamaguchi T, Takashima A, Nagashima K, Makuuchi R, Aizawa M, Ohashi M, et al. Efficacy of Postoperative Chemotherapy After Resection that Leaves No Macroscopically Visible Disease of Gastric Cancer with Positive Peritoneal Lavage Cytology (CY1) or Localized Peritoneum Metastasis (P1a): A Multicenter Retrospective Study. Annals of surgical oncology. 2020; 27: 284-92.

16. Yang S, Feng R, Pan ZC, Jiang T, Xu Q, Chen Q. A Comparison of Intravenous plus Intraperitoneal Chemotherapy with Intravenous Chemotherapy Alone for the Treatment of Gastric Cancer: A Meta-Analysis. Scientific reports. 2015; 5: 12538.

17. Ishigami H, Fujiwara Y, Fukushima R, Nashimoto A, Yabusaki H, Imano M, et al. Phase III Trial Comparing Intraperitoneal and Intravenous Paclitaxel Plus S-1 Versus Cisplatin Plus S-1 in Patients with Gastric Cancer with Peritoneal Metastasis: PHOENIX-GC Trial. Journal of clinical oncology: official journal of the American Society of Clinical Oncology. 2018; 36: 1922-9.

18. Yamaguchi $\mathrm{H}$, Kitayama J, Ishigami H, Kazama S, Nozawa H, Kawai K, et al. Breakthrough therapy for peritoneal carcinomatosis of gastric cancer: Intraperitoneal chemotherapy with taxanes. World journal of gastrointestinal oncology. 2015; 7: 285-91.

19. Deng K, Zhu H, Chen M, Wu J, Hu R, Tang C. Prognostic Significance of Molecular Analysis of Peritoneal Fluid for Patients with Gastric Cancer: A Meta-Analysis. PloS one. 2016; 11: e0151608.

20. Virgilio E, Giarnieri E, Giovagnoli MR, Montagnini M, Proietti A, D'Urso R, et al. Gastric Cancer Cells in Peritoneal Lavage Fluid: A Systematic Review Comparing Cytological with Molecular Detection for Diagnosis of Peritoneal Metastases and Prediction of Peritoneal Recurrences. Anticancer research. 2018; 38: 1255-62.

21. Jeon $\mathrm{CH}, \mathrm{Kim} \mathrm{IH}$, Chae HD. Prognostic value of genetic detection using CEA and MAGE in peritoneal washes with gastric carcinoma after curative resection: result of a 3-year follow-up. Medicine. 2014; 93: e83.

22. Li Z, Zhang D, Zhang H, Miao Z, Tang Y, Sun G, et al. Prediction of peritoneal recurrence by the mRNA level of CEA and MMP-7 in peritoneal lavage of gastric cancer patients. Tumour biology: the journal of the International Society for Oncodevelopmental Biology and Medicine. 2014; 35: 3463-70.

23. Laghi A, Bellini D, Rengo M, Accarpio F, Caruso D, Biacchi D, et al. Diagnostic performance of computed tomography and magnetic resonance imaging for detecting peritoneal metastases: systematic review and meta-analysis. Radiol Med. 2017; 122: 1-15. 